\title{
Exponents of Diophantine approximation and expansions in integer bases
}

\author{
Masaaki Amou (Kiryu) \& Yann Bugeaud (Strasbourg)
}

\begin{abstract}
Let $\xi$ be a real number and $b \geq 2$ be an integer. Let $v_{b}(\xi)$ (resp. $v_{b}^{\prime}(\xi)$ ) denote the supremum of the real numbers $v$ for which the equation $\left\|b^{n} \xi\right\| \leq\left(b^{n}\right)^{-v}$ (resp. $\left\|b^{r}\left(b^{s}-1\right) \xi\right\| \leq\left(b^{r+s}\right)^{-v}$ ) has infinitely many solutions in positive integers $n$ (resp. $r$ and $s$ ). Here, $\|\cdot\|$ stands for the distance to the nearest integer. Let also $v_{1}(\xi)$ denote the supremum of the real numbers $v$ for which the equation $\|q \xi\|<q^{-v}$ has infinitely many solutions in positive integers $q$. Motivated by the question whether one can read the irrationality exponent of a real number on its b-ary expansion, we establish various results on the set of values taken by the triple of functions $\left(v_{1}, v_{b}, v_{b}^{\prime}\right)$ evaluated at real points.
\end{abstract}

\section{Introduction}

Let $\xi$ be an irrational real number. For convenience, we assume that $0<\xi<1$. The irrationality exponent of $\xi$, commonly denoted by $\mu(\xi)$, is the supremum of the real numbers $\mu$ for which

$$
0<\left|\xi-\frac{p}{q}\right|<\frac{1}{q^{\mu}}
$$

has infinitely many rational solutions $p / q$. It follows from the theory of continued fractions (or from Dirichlet's Schubfachprinzip) that $\mu(\xi)$ is at least equal to 2 . In other words, there are infinitely many rational numbers that approximate $\xi$ at order at least two. Moreover, $\mu(\xi)$ is exactly equal to two for almost all real numbers $\xi$. Here and below, 'almost all' always refers to the Lebesgue measure.

A very naïve way to produce good rational approximations to $\xi$ is to write $\xi$ in some integer base $b$, with $b \geq 2$, that is to consider its expansion

$$
\xi=\sum_{j \geq 1} \frac{a_{j}}{b^{j}},
$$

2000 Mathematics Subject Classification : 11J04. 
where $a_{j} \in\{0,1, \ldots, b-1\}$ for $j \geq 1$. If $a_{J+1}=\ldots=a_{J+r}=0$ for some integers $J, r$, then $\xi$ is quite close to the rational number $\sum_{j=1}^{J} a_{j} / b^{j}$, namely

$$
\left|\xi-\sum_{j=1}^{J} \frac{a_{j}}{b^{j}}\right|<\frac{1}{b^{J+r}} .
$$

However, a classical argument based on the Cantelli lemma shows us that almost all real numbers have only finitely many very large blocks of zeros occurring near the beginning of their $b$-ary expansion. More precisely, for any $\varepsilon>0$ and almost all real numbers $\xi$, there are only finitely many pairs $\left(J, p_{J}\right)$ of integers such that $\left|\xi-p_{J} / b^{J}\right|<\left(b^{J}\right)^{-1-\varepsilon}$. Consequently, this very naïve approach based on the $b$-ary expansion only yields that the irrationality exponent of $\xi$ is at least equal to 1 , which is a trivial result.

A slightly less naïve method to construct good rational approximations to $\xi$ consists in locating periodic motives in its $b$-ary expansion (1.1), that is, in finding integers $J, r, m$ such that $a_{J+h+i r}=a_{J+h}$ for $h=1, \ldots, r$ and $i=1, \ldots, m$. With such a triple $(J, r, m)$ of integers, we associate the rational number $\xi_{J}$ whose $b$-ary expansion is defined as follows: Truncate the expansion of $\xi$ after the $J$-th digit $a_{J}$ and complete by repeating infinitely many copies of the finite word $a_{J+1} \ldots a_{J+r}$. Clearly, $\xi_{J}$ is a good rational approximation to $\xi$ which satisfies

$$
\left|\xi-\xi_{J}\right|<\frac{1}{b^{J+(m+1) r}} .
$$

The same argument as above shows us that almost all real numbers have only finitely many very large repetitions occurring near the beginning of their $b$-ary expansion, and this slightly less naïve approach only yields that the irrationality exponent of $\xi$ is at least equal to 1 , which is a trivial result.

This short discussion shows that, apparently, there is in general no relation between the $b$-ary expansion of a number and its irrationality exponent. Thus, the answer to the following question, that motivates the present note, is essentially negative:

Can one read the irrationality exponent of a real number on its expansion in some integer base?

To investigate more closely this question, we are led to introduce new exponents of approximation. Throughout the present paper, $\|\cdot\|$ denotes the distance to the nearest integer and $\lfloor\cdot\rfloor$ stands for the integer part function.

Definition 1. Let $\xi$ be an irrational real number. Let $b$ be an integer with $b \geq 2$. We denote by $v_{b}(\xi)$ the supremum of the real numbers $v$ for which the equation

$$
\left\|b^{n} \xi\right\|<\left(b^{n}\right)^{-v}
$$

has infinitely many solutions in positive integers $n$. We denote by $v_{b}^{\prime}(\xi)$ the supremum of the real numbers $v$ for which the equation

$$
\left\|b^{r}\left(b^{s}-1\right) \xi\right\|<\left(b^{r+s}\right)^{-v}
$$


has infinitely many solutions in positive integers $r$ and $s$.

The exponents $v_{b}^{\prime}$ are closely related to the Diophantine exponent of an infinite word introduced in [1].

For consistency, we denote by $v_{1}(\xi)$ the supremum of the real numbers $v$ for which the equation

$$
\|q \xi\|<q^{-v}
$$

has infinitely many solutions in positive integers $q$. The exponent $v_{1}$, usually denoted by $w_{1}$, is equal to the irrationality exponent decreased by 1 . Jarník [15] established that the function $v_{1}$ takes every value between 1 and $+\infty$. As already noticed, $v_{1}(\xi)=1$ and $v_{b}^{\prime}(\xi)=v_{b}(\xi)=0$ hold for every integer $b \geq 2$ and for almost all real numbers $\xi$.

Let $\xi$ be an irrational number. We have $v_{b}^{\prime}(\xi) \geq v_{b}(\xi) \geq 0$ for $b \geq 2$, and

$$
v_{1}(\xi) \geq \max \left\{v_{b}^{\prime}(\xi), 1\right\} \geq \max \left\{v_{b}(\xi), 1\right\}, \quad \text { for } b \geq 2 .
$$

Furthermore, it is not difficult to check that

$$
v_{b}(\xi)=v_{b^{t}}(\xi), \quad \text { and } \quad v_{b}^{\prime}(\xi)=v_{b^{t}}^{\prime}(\xi), \quad \text { for } t \text { in } \mathbf{Z}_{\geq 1} \text { and } b \geq 2 .
$$

Moreover, the value $v_{b_{2}}(\xi)$ is related to the value $v_{b_{1}}(\xi)$ if the set of prime factors of $b_{1}$ is a subset of the set of prime factors of $b_{2}$.

To see this, let $m_{1}$ and $m_{2}$ be positive integers such that $b_{1}^{m_{1}}$ divides $b_{2}^{m_{2}}$. Let $\varepsilon$ be a positive real number. Then there exist arbitrarily large integers $n$ such that

$$
\left\|b_{1}^{n} \xi\right\|<\left(b_{1}^{n}\right)^{-v+\varepsilon}, \quad v=v_{b_{1}}(\xi) .
$$

Setting $n=(k-1) m_{1}+r$ with $0 \leq r<m_{1}$, we have

$$
\left\|b_{2}^{m_{2} k} \xi\right\|<\left(b_{1}\right)^{\left(m_{1}-r\right)(v+1-\varepsilon)}\left(b_{2}^{m_{2}} / b_{1}^{m_{1}}\right)^{k}\left(b_{1}^{m_{1} k}\right)^{-v+\varepsilon},
$$

which implies that

$$
v_{b_{2}}(\xi)+1 \geq \frac{m_{1} \log b_{1}}{m_{2} \log b_{2}}\left(v_{b_{1}}(\xi)+1\right)
$$

Hence, we have

$$
v_{b_{2}}(\xi)+1 \geq\left(\sup _{m_{1}, m_{2}: b_{1}^{m_{1}} \mid b_{2}^{m_{2}}} \frac{m_{1}}{m_{2}}\right) \frac{\log b_{1}}{\log b_{2}}\left(v_{b_{1}}(\xi)+1\right),
$$

where, as indicated, the supremum ranges over all positive integers $m_{1}, m_{2}$ such that $b_{1}^{m_{1}}$ divides $b_{2}^{m_{2}}$.

This supremum can be easily expressed in terms of $b_{1}$ and $b_{2}$. To see this, write

$$
b_{1}=p_{1}^{e_{1}} \ldots p_{\ell}^{e_{\ell}}, \quad b_{2}=p_{1}^{f_{1}} \ldots p_{\ell}^{f_{\ell}} \cdot b_{2}^{\prime},
$$

where $p_{1}, \ldots, p_{\ell}$ are distinct prime numbers, $e_{i}, f_{i}$ are positive integers and $b_{2}^{\prime}$ is coprime to $p_{1} \ldots p_{\ell}$. Since $b_{1}^{m_{1}}$ divides $b_{2}^{m_{2}}$ if and only if $m_{1} e_{i} \leq m_{2} f_{i}$ for all $i$, we deduce that

$$
\sup _{m_{1}, m_{2}: b_{1}^{m_{1}} \mid b_{2}^{m_{2}}} \frac{m_{1}}{m_{2}}=\min _{1 \leq i \leq \ell} \frac{f_{i}}{e_{i}},
$$

which leads us to define

$$
\rho\left(b_{1}, b_{2}\right):=\min _{1 \leq i \leq \ell} \frac{f_{i}}{e_{i}}
$$

Denoting by $\mathcal{P}(b)$ the set of prime factors of an integer $b \geq 2$, we have established the following fact. 
Fact. For every real irrational number $\xi$, for every integers $b_{1}, b_{2}$ satisfying $b_{1} \geq 2, b_{2} \geq 2$ and $\mathcal{P}\left(b_{1}\right) \subseteq \mathcal{P}\left(b_{2}\right)$ we have

$$
v_{b_{2}}(\xi)+1 \geq \rho\left(b_{1}, b_{2}\right) \frac{\log b_{1}}{\log b_{2}}\left(v_{b_{1}}(\xi)+1\right) .
$$

Throughout this text, we denote by $\mathcal{B}$ the set consisting of all positive integers which are not perfect powers, thus $\mathcal{B}=\{2,3,5,6,7,10, \ldots\}$, and we set $\mathcal{B}_{1}=\{1\} \cup \mathcal{B}$.

We are mainly concern with the following two questions.

Problem 1. Let $\left(v_{b}\right)_{b \in \mathcal{B}_{1}}$ and $\left(v_{b}^{\prime}\right)_{b \in \mathcal{B}}$ be sequences of real numbers or $+\infty$ satisfying

$$
v_{1} \geq 1, \quad 0 \leq v_{b} \leq v_{b}^{\prime} \leq v_{1}, \quad \text { for every } b \in \mathcal{B},
$$

and

$$
v_{b_{2}}+1 \geq \rho\left(b_{1}, b_{2}\right) \frac{\log b_{1}}{\log b_{2}}\left(v_{b_{1}}+1\right), \quad \text { for every } b_{1}, b_{2} \in \mathcal{B} \text { such that } \mathcal{P}\left(b_{1}\right) \subseteq \mathcal{P}\left(b_{2}\right) .
$$

To prove that there exist real numbers $\xi$ such that

$$
v_{1}(\xi)=v_{1}, \quad v_{b}(\xi)=v_{b} \quad \text { and } \quad v_{b}^{\prime}(\xi)=v_{b}^{\prime}, \quad \text { for every } b \in \mathcal{B}
$$

Problem 1 in its full generality seems to be quite out of reach. More modestly, we also focus on the following question.

Problem 2. Let $b \geq 2$ be an integer. Let $v_{1}, v_{b}, v_{b}^{\prime}$ be elements of $\mathbf{R} \cup\{+\infty\}$ with

$$
v_{1} \geq 1, \quad 0 \leq v_{b} \leq v_{b}^{\prime} \leq v_{1} .
$$

To prove that there exist real numbers $\xi$ with

$$
v_{1}(\xi)=v_{1}, \quad v_{b}(\xi)=v_{b} \quad \text { and } \quad v_{b}^{\prime}(\xi)=v_{b}^{\prime}
$$

In other words, a base $b$ being given (which is not assumed to be in $\mathcal{B}$ ), Problem 2 asks for the joint spectrum of the triple of functions $\left(v_{1}, v_{b}, v_{b}^{\prime}\right)$. Problems 1 and 2 should be compared with the Main Problem studied in [7], where, besides rational approximation, the quality of approximation by algebraic numbers of bounded degree is also considered. It is a well-known fact that most difficulties arise for determining the small values of the spectrum, see the discussion in Section 7.8 of [7].

The present paper is organized as follows. Our main contributions towards Problems 1 and 2 are stated in Section 2 and established in Sections 3 to 7. One key tool is a version of the Folding Lemma for continued fractions given in Section 4. In Section 8, we use our version of the Folding Lemma to construct explicitly real numbers with prescribed irrationality exponent that are normal in a given base.

\section{Main results}

We begin with a general result that solves partially Problem 1. 
Theorem 1. Let $\left(v_{b}\right)_{b \in \mathcal{B}_{1}}$ be a sequence of elements of $\mathbf{R} \cup\{+\infty\}$ satisfying

$$
\frac{1+\sqrt{5}}{2} \leq v_{b} \leq v_{1}, \quad \text { for every } b \in \mathcal{B},
$$

and

$$
v_{b_{2}}+1 \geq \rho\left(b_{1}, b_{2}\right) \frac{\log b_{1}}{\log b_{2}}\left(v_{b_{1}}+1\right), \quad \text { for every } b_{1}, b_{2} \in \mathcal{B} \text { such that } \mathcal{P}\left(b_{1}\right) \subseteq \mathcal{P}\left(b_{2}\right) .
$$

There exist uncountably many real numbers $\xi$ such that

$$
v_{b}(\xi)=v_{b}, \quad \text { for every } b \in \mathcal{B}_{1} \text {. }
$$

The proof of Theorem 1 is postponed to Section 3. The idea is the following: We construct real numbers $\xi$ together with all the rational numbers $p / q$ such that $|\xi-p / q| \ll$ $q^{-(3+\sqrt{5}) / 2}$. Here and throughout the present paper, the notation $\ll$ and $\gg$ means that some absolute positive numerical constant is implicit. If $A \ll B$ and $A \gg B$ hold simultaneously, then we write $A \asymp B$. The main tool for computing the exact values of the various exponents of approximation is then simply triangle inequalities. The lower bound $(1+\sqrt{5}) / 2$ occurring in $(2.1)$ is therefore not surprising. It already appeared in earlier works $[7,18]$ and may be briefly explained as follows. Let $\xi$ be a real number and $p / q$ and $p^{\prime} / q^{\prime}$ be two rational numbers with

$$
|\xi-p / q| \asymp q^{-\tau}, \quad\left|\xi-p^{\prime} / q^{\prime}\right| \asymp q^{\prime-\tau}, \quad \text { and } \quad q^{\prime} \asymp q^{\tau},
$$

for some real number $\tau \geq 1$. To guarantee that no rational number $a / b$ with $q<b<q^{\prime}$ approximates $\xi$ at an order greater than $\tau$, we argue as follows (the correctness of the argument depends on the hidden constant if $\left.b \asymp q^{\tau-1}\right)$. For $b \ll q^{\tau-1}$, we note that

$$
\left|\xi-\frac{a}{b}\right| \gg\left|\frac{p}{q}-\frac{a}{b}\right|-\left|\xi-\frac{p}{q}\right| \gg \frac{1}{q^{\tau}} \gg \frac{1}{b^{\tau}},
$$

while, for $b \gg q^{1 /(\tau-1)}$, we observe that

$$
\left|\xi-\frac{a}{b}\right| \gg\left|\frac{p^{\prime}}{q^{\prime}}-\frac{a}{b}\right|-\left|\xi-\frac{p^{\prime}}{q^{\prime}}\right| \gg \frac{1}{b q^{\prime}} \gg \frac{1}{b^{\tau}} .
$$

To cover all the interval $q<b<q^{\prime}$, we need to have $q^{1 /(\tau-1)} \ll q^{\tau-1}$, that is $\tau \geq$ $(3+\sqrt{5}) / 2$. If $\tau$ is smaller, we cannot exclude that there are better approximations to $\xi$ with a denominator between $q$ and $q^{\prime}$.

We can go through the proof of Theorem 1 with sequences that do not satisfy (2.1). Our construction would then even yield the existence of real numbers $\xi$ such that

$$
v_{1}(\xi) \geq v_{1}, \quad v_{b}(\xi) \geq v_{b} \quad \text { and } \quad v_{b}^{\prime}(\xi) \geq v_{b}^{\prime}, \quad \text { for every } b \in \mathcal{B} .
$$

We will not be able to show that these inequalities are indeed equalities, since we are not certain to know all the best rational approximations to $\xi$.

One way to overcome this difficulty is to construct simultaneously the continued fraction expansion of $\xi$ and its expansion in base $b$. This was already done in [9] to solve a problem of Mahler on rational approximation to elements of Cantor sets. By means of a suitable extension of a lemma of Amou [2] and using ideas of Mahler [19, 20, 21], we establish the following significant partial result on Problem 2. 
Theorem 2. Let $b \geq 2$ be an integer. Let $v_{1}, v_{b}, v_{b}^{\prime}$ be elements of $\mathbf{R} \cup\{+\infty\}$ with

$$
v_{1} \geq 1, \quad 1 \leq v_{b} \leq v_{b}^{\prime} \leq v_{1}
$$

or

$$
v_{1} \geq 1, \quad v_{b}=0, \quad \text { and either } \quad v_{b}^{\prime}=0, \quad \text { or } \quad 1 \leq v_{b}^{\prime} \leq v_{1} .
$$

There exist uncountably many real numbers $\xi$ with

$$
v_{1}(\xi)=v_{1}, \quad v_{b}(\xi)=v_{b} \quad \text { and } \quad v_{b}^{\prime}(\xi)=v_{b}^{\prime}
$$

To point out the main ideas of its proof, Theorem 2 is established step by step. We begin (see Theorem 5 in Section 4 ) by the case where $v_{1}=v_{b}=v_{b}^{\prime}$. Then, in the subsequent section, we treat the case where $v_{1} \geq b-1$ and $v_{b}=v_{b}^{\prime}=0$ (see Theorem 6 ). The remaining steps are explained in Section 5.

Unfortunately, Theorem 2 gives nothing when one of the prescribed values $v_{b}, v_{b}^{\prime}$ is strictly between 0 and 1 . To tackle this case, we can use again triangle inequalities, but we need then to assume that $v_{1}$ is sufficiently large.

Theorem 3. Let $b \geq 2$ be an integer. Let $v_{1}, v_{b}$ and $v_{b}^{\prime}$ be real numbers such that

$$
0 \leq v_{b} \leq v_{b}^{\prime} \leq \frac{v_{b}^{\prime}+1+\sqrt{\left(v_{b}^{\prime}\right)^{2}+6 v_{b}^{\prime}+5}}{2} \leq v_{1} .
$$

Then, there exist uncountably many real numbers $\xi$ such that

$$
v_{b}(\xi)=v_{b}, \quad v_{b}^{\prime}(\xi)=v_{b}^{\prime} \quad \text { and } \quad v_{1}(\xi)=v_{1} .
$$

Theorem 3 is proved in Section 6, where we mention another similar partial result on Problem 2 that can be established following the same method.

We end this section with a further (small) contribution towards the resolution of Problem 1.

Theorem 4. For any non-negative real number $v$, there exist uncountably many real numbers $\xi$ such that

$$
v_{1}(\xi)=2 v+1
$$

and

$$
v_{b}(\xi)=v_{b}^{\prime}(\xi)=v, \quad \text { for every } b \geq 2 .
$$

The proof of Theorem 4, outlined in Section 7, uses methods from metric number theory.

Theorem 4 may be compared with Theorem 5.8 from [7], where the theory of intersective sets is applied to other exponents of approximation that generalize the exponent $v_{1}$.

\section{On explicit constructions: continued fraction expansions}

The proof of Theorem 1 rests on the following lemma. 
Lemma 1. Let $b \geq 2$ be an integer and $\mu \geq(3+\sqrt{5}) / 2$ be a real number. Let $m$ be a positive integer. Let $P / Q$ and $R / S$ be reduced fractions with positive denominators such that

$$
S^{\mu} \leq Q<b S^{\mu}
$$

and

$$
\frac{1}{Q} \leq\left|\frac{P}{Q}-\frac{R}{S}\right| \leq \frac{m}{Q}
$$

Then, for any reduced fraction $A / B$ with $S \leq B<Q$, we have

$$
\left|\frac{P}{Q}-\frac{A}{B}\right|>\frac{1}{b(2 m)^{1.7} B^{\mu}} .
$$

Proof. Assume first that $S \leq B \leq S^{\mu-1} /(2 m)$. Since this with (3.1) gives $B S \leq$ $S^{\mu} /(2 m) \leq Q /(2 m)$, using

$$
\left|\frac{P}{Q}-\frac{A}{B}\right| \geq\left|\frac{A}{B}-\frac{R}{S}\right|-\left|\frac{P}{Q}-\frac{R}{S}\right|
$$

together with (3.2), we obtain

$$
\left|\frac{P}{Q}-\frac{A}{B}\right| \geq \frac{1}{B S}-\frac{m}{Q} \geq \frac{1}{2 B S} \geq \frac{1}{2 B^{2}} .
$$

Assume next that $S^{\mu-1} /(2 m)<B<Q$. Since this with (3.1) gives $Q<b(2 m B)^{\mu /(\mu-1)}$, we obtain

$$
\left|\frac{P}{Q}-\frac{A}{B}\right| \geq \frac{1}{B Q}>\frac{1}{b B(2 m B)^{\mu /(\mu-1)}},
$$

which implies (3.3), since $\mu /(\mu-1)<1.7$ and $1+\mu /(\mu-1) \leq \mu$. Thus the lemma is proved.

Remark. In Lemma 1 , if $m / S^{\mu}<1 /\left(2 S^{2}\right)$, that is, if $S>(2 m)^{1 /(\mu-2)}$, then $R / S$ is a convergent to $P / Q$. This is in particular the case if $S>(2 m)^{2}$.

Proof of Theorem 1. Let us take a sequence $\left(b_{j}\right)_{j \geq 1}$ with $b_{j} \in \mathcal{B}_{1}$ such that $b_{1}=1, b_{j} \leq j$ and, for every $b \in \mathcal{B}_{1}$, there are infinitely many $j$ satisfying $b_{j}=b$. Let $R_{0} / S_{0}$ be a reduced fraction with $S_{0}>100$. Assume that we have already constructed reduced fractions $P_{i} / Q_{i}$ and $R_{i} / S_{i}$ for $i=1, \ldots, j-1$ with $j \in \mathbf{N}$. (Note that we do not have $P_{0} / Q_{0}$.) Then we construct inductively reduced fractions $P_{j} / Q_{j}$ and $R_{j} / S_{j}$ as follows.

We first take $Q_{j}=b_{j}^{n_{j}}$ (resp. $Q_{j}$ prime) with $n_{j} \geq 1$ if $b_{j} \geq 2$ (resp. if $b_{j}=1$ ) such that the triple $(S, Q, b)=\left(S_{j-1}, Q_{j}, b_{j}\right)\left(\right.$ resp. $\left.(S, Q, b)=\left(S_{j-1}, Q_{j}, 2\right)\right)$ satisfies (3.1) with $\mu=(3+\sqrt{5}) / 2$, and next take an integer $P_{j}$ such that $P_{j} / Q_{j}$ is reduced and that the pair $(R / S, P / Q)=\left(R_{j-1} / S_{j-1}, P_{j} / Q_{j}\right)$ satisfies (3.2) with $m=m(b):=2 b+2$. Denoting a continued fraction expansion of $P_{j} / Q_{j}$ by $\left[a_{0} ; a_{1}, \ldots, a_{k}\right]$, we define a reduced fraction $R_{j} / S_{j}$ by

$$
\frac{R_{j}}{S_{j}}:=\left[a_{0} ; a_{1}, \ldots, a_{k},\left\lfloor Q_{j}^{v-1}\right\rfloor\right]
$$


where $v=v_{b_{j}}$ if $v_{b_{j}}<+\infty$ and $v=j$ if $v_{b_{j}}=+\infty$.

By the construction we can define a real number $\xi$ by

$$
\xi:=\lim _{j \rightarrow+\infty} \frac{P_{j}}{Q_{j}},
$$

whose continued fraction expansion has $P_{j} / Q_{j}$ and $R_{j} / S_{j}(j \in \mathbf{N})$ among its convergents. We claim that this $\xi$ satisfies the conditions given in the theorem. To this end we consider rational approximations to $\xi$.

Let $j$ be a positive integer and set $v=v_{b_{j}}$. Under the above notation, since

$$
\left[a_{0} ; a_{1}, \ldots, a_{k},\left\lfloor Q_{j}^{v-1}\right\rfloor-1\right]
$$

is the only possible convergent to $\xi$ between $P_{j} / Q_{j}$ and $R_{j} / S_{j}$, we have

$$
\frac{1}{Q_{j}\left(S_{j}+Q_{j}\right)}<\left|\xi-\frac{P_{j}}{Q_{j}}\right|<\frac{1}{Q_{j}\left(S_{j}-Q_{j}\right)},
$$

which implies

$$
\frac{1}{2 Q_{j}^{v+1}}<\left|\xi-\frac{P_{j}}{Q_{j}}\right|<\frac{2}{Q_{j}^{v+1}} .
$$

For the same reason, for any reduced fraction $A / B$ with $Q_{j}<B<S_{j}(j \in \mathbf{N})$, we have

$$
\left|\xi-\frac{A}{B}\right| \geq \frac{1}{2 B^{2}} .
$$

Let $A / B$ be a reduced fraction with $S_{j-1} \leq B<Q_{j}(j \in \mathbf{N})$. Since

$$
\left|\frac{P_{j}}{Q_{j}}-\frac{A}{B}\right| \geq \frac{1}{B Q_{j}}, \quad\left|\xi-\frac{P_{j}}{Q_{j}}\right| \leq \frac{2}{Q_{j}^{v+1}},
$$

and since $B Q_{j}<Q_{j}^{v+1} / 4$, we have

$$
\left|\xi-\frac{A}{B}\right| \geq\left|\frac{P_{j}}{Q_{j}}-\frac{A}{B}\right|-\left|\xi-\frac{P_{j}}{Q_{j}}\right|>\frac{1}{2}\left|\frac{P_{j}}{Q_{j}}-\frac{A}{B}\right| .
$$

We then infer from Lemma 1 that

$$
\left|\xi-\frac{A}{B}\right|>\frac{1}{2 b(2 m(b))^{1.7} B^{\mu}},
$$

where $b=b_{j}$ and $m(b)=2 b+2$.

Consequently, on noting that $b_{j}=O(j)$, these estimates prove our claim.

In each step of the inductive procedure, there are at least two choices of $P_{j} / Q_{j}$ having the same denominator $Q_{j}$; one is less than $R_{j-1} / S_{j-1}$, and the other is greater than 
$R_{j-1} / S_{j-1}$. Hence we have an infinite directed binary tree of reduced fractions whose infinite paths correspond to real numbers $\xi$, which are different from each other and have the conditions given in the assertion. This ensures the uncountability of the desired numbers, and completes the proof of the theorem.

For sake of clarity, we add a few words on $(2.2)$. Let $b$ and $b^{\prime}$ be distinct elements of $\mathcal{B}$ such that $\mathcal{P}\left(b^{\prime}\right) \subseteq \mathcal{P}(b)$. Then, for any $Q_{j}^{\prime}=\left(b^{\prime}\right)^{n^{\prime}}$ with some positive integer $n^{\prime}$, we have to estimate from below

$$
\left|\xi-\frac{P_{j}^{\prime}}{Q_{j}^{\prime}}\right|=\left|\xi-\frac{P_{j}}{Q_{j}}\right|
$$

with $Q_{j}=b^{n}$ and $P_{j}=P_{j}^{\prime}\left(Q_{j} / Q_{j}^{\prime}\right)$ provided that $Q_{j}^{\prime}$ divides $Q_{j}$. Since, by $(3.4)$,

$$
\left|\xi-\frac{P_{j}^{\prime}}{Q_{j}^{\prime}}\right|>\frac{1}{2\left(Q_{j}^{\prime}\right)^{v_{b^{\prime}}+1}},
$$

we have

$$
\left|\xi-\frac{P_{j}}{Q_{j}}\right|>\frac{1}{2}\left(Q_{j}\right)^{-\frac{n^{\prime} \log b^{\prime}}{n \log b}\left(v_{b^{\prime}}+1\right)} .
$$

On noting that $n^{\prime} / n \leq \rho\left(b^{\prime}, b\right)$, we get

$$
\left|\xi-\frac{P_{j}}{Q_{j}}\right|>\frac{1}{2}\left(Q_{j}\right)^{-\rho\left(b^{\prime}, b\right) \frac{\log b^{\prime}}{\log b}\left(v_{b^{\prime}}+1\right)} .
$$

In view of the assumption (2.2), this shows that $v_{b}(\xi)=v_{b}$. To see this, one should add that, if $j$ is such that $b_{j}=1$, then, by assumption, $Q_{j}$ is a prime number and there are at most finitely many such $j$ for which $\mathcal{P}\left(Q_{j}\right)$ is contained in $\mathcal{P}(b)$.

To conclude this section, we explain why the approach followed in the proof of Theorem 1 cannot be applied to construct real numbers $\xi$ with prescribed values for $v_{b}^{\prime}(\xi)$, where $b \in \mathcal{B}$. The point is that we do not control all the rational approximations that give the value of $v_{b}^{\prime}(\xi)$. Indeed, with the above notation, let us consider the fractions $P_{j} / Q_{j}$ for the indices $j$ with $b_{j}=1$. Let $b$ be in $\mathcal{B}$. Then, there exist integers $T_{j}, r_{j}$ and $s_{j}$ such that

$$
\frac{P_{j}}{Q_{j}}=\frac{T_{j}}{b^{r_{j}}\left(b^{s_{j}}-1\right)},
$$

where the latter fraction may not be written in reduced form. It may happen that $b^{r_{j}}\left(b^{s_{j}}-\right.$ 1 ) is not much greater than $Q_{j}$. If this is the case for infinitely many $j$, we may even get that $v_{b}^{\prime}(\xi)=v_{1}(\xi)$. Since there are no ways to control $r_{j}$ and $s_{j}$, we cannot get the exact value of $v_{b}^{\prime}(\xi)$.

\section{On explicit constructions: the Folding Lemma}

Our goal in this section is to describe a general method for constructing numbers $\xi$ with prescribed exponents $v_{1}(\xi)$ and $v_{b}(\xi)$ for some integer $b \geq 2$. Since the value of $v_{1}(\xi)$ can be read on the continued fraction expansion of $\xi$, we aim at constructing real numbers

whose continued fraction and $b$-ary expansions are simultaneously explicitly known. The key tool for this is the Folding Lemma [23, 24, 25]. 
Lemma $\mathbf{F}$. Let $n, a_{1}, \ldots, a_{n}$ be positive integers. Let $t \geq 2$ be an integer. If $p_{n} / q_{n}=$ $\left[a_{0} ; a_{1}, a_{2}, \ldots, a_{n}\right]$ with $a_{n} \geq 2$, then

$$
\frac{p_{n}}{q_{n}}+\frac{(-1)^{n}}{t q_{n}^{2}}=\left[a_{0} ; a_{1}, a_{2}, \ldots, a_{n-1}, a_{n}, t-1,1, a_{n}-1, a_{n-1}, \ldots, a_{2}, a_{1}\right] .
$$

We display an immediate consequence of Lemma F.

Lemma 2. Let $t \geq 2$ be an integer. If $a / m=\left[0 ; 1,1, a_{3}, \ldots, a_{h-1}, a_{h}\right]$ with $h \geq 4$ and $a_{h} \geq 2$, then

$$
\frac{t m a+(-1)^{h}}{t m^{2}}=\left[0 ; 1,1, a_{3}, \ldots, a_{h-1}, a_{h}, t-1,1, a_{h}-1, a_{h-1}, \ldots, a_{3}, 2\right]
$$

Lemma 2 is the main tool for the proof of Theorem 5 below, which settles a particular case of Theorem 2 .

Theorem 5. Let $b \geq 2$ be an integer. For any real number $v>1$, there exist uncountably many real numbers $\xi$ such that

$$
v_{1}(\xi)=v_{b}(\xi)=v_{b}^{\prime}(\xi)=v
$$

Proof. Observe that in the open real interval with endpoints $[0 ; 1,1,1,2]$ and $[0 ; 1,1,1,3]$ there are rational numbers whose denominator is a power of $b$. Consequently, there exist positive integers $a$ and $\ell$, with $1 \leq a \leq b^{\ell}$ and $a$ coprime with $b$, such that the continued fraction of $a / b^{\ell}$ reads

$$
\frac{a}{b^{\ell}}=\left[0 ; 1,1, a_{3}, \ldots, a_{h-1}, a_{h}\right]
$$

with $h \geq 4$ and $a_{h} \geq 2$.

Let $\left(u_{n}\right)_{n \geq 1}$ be a sequence of positive integers. Applying Lemma 2 first with $t=b^{u_{1}}$, then with $t=\bar{b}^{u_{2}}$, and so on, we obtain that the real number

$$
\xi=\frac{a}{b^{\ell}} \pm \frac{1}{b^{u_{1}+2 \ell}}-\frac{1}{b^{u_{2}+2 u_{1}+4 \ell}}-\ldots-\frac{1}{b^{u_{k}+2 u_{k-1}+\ldots+2^{k-1} u_{1}+2^{k} \ell}}-\ldots
$$

satisfies

$$
\left|\xi-\frac{p_{k}}{b^{u_{k}+2 u_{k-1}+\ldots+2^{k-1} u_{1}+2^{k} \ell}}\right| \asymp \frac{1}{b^{u_{k+1}+2\left(u_{k}+2 u_{k-1}+\ldots+2^{k-1} u_{1}+2^{k} \ell\right)}}, \quad \text { for } k \geq 1
$$

where $p_{k}$ is the closest integer to $\left(b^{u_{k}+2 u_{k-1}+\ldots+2^{k-1} u_{1}+2^{k} \ell}\right) \xi$. These rational approximations to $\xi$ are the best possible because $b^{u_{k+1}}-1$ is the largest partial quotient at the beginning of the continued fraction expansion of $\xi$.

Let $v>1$ be given. Choose inductively $u_{k}$ such that

$$
u_{k+1} \asymp(v-1)\left(u_{k}+2 u_{k-1}+\ldots+2^{k-1} u_{1}+2^{k} \ell\right) .
$$


It is sufficient to take $u_{1}=\lfloor(v-1) \ell\rfloor$ and

$$
u_{k+1}=\left\lfloor(v-1)\left(u_{k}+\ldots+2^{k} \ell\right)\right\rfloor, \quad \text { for } k \geq 1 \text {. }
$$

We are done, since we perfectly control the best rational approximations. We obtain at once that

$$
v_{b}(\xi)=v \quad \text { and } \quad v_{1}(\xi)=v
$$

This completes the proof of the theorem.

Theorem 5 will be considerably improved upon in the next section, thanks to the following far-reaching extension of Lemma 2 that takes its source in [2].

Lemma 3. Let $\tau$ be a positive number with $\tau \geq 2$. Let $\left(A_{n}\right)_{n \geq 1}$ be a strictly increasing sequence of positive integers and $\left(B_{n}\right)_{n \geq 1}$ be a sequence of nonzero integers. For $n \geq 1$, set

$$
S_{n}=\left(A_{n+1} /\left|B_{n+1}\right|\right) /\left(A_{n} /\left|B_{n}\right|\right)^{2}
$$

and denote by $d_{n}$ the denominator of $S_{n}$. Furthermore, we set

$$
D_{n}=\prod_{\nu=0}^{n} d_{\nu}^{2^{n-\nu}}
$$

for $n \geq 0$, where $d_{0}=\left|B_{1}\right|$. Assume that the following conditions are satisfied:

(i) $S_{n} \geq 2$ holds for any sufficiently large $n$;

(ii) $\limsup _{n \rightarrow \infty} \frac{\log A_{n+1}}{\log A_{n}}=\tau$;

(iii) $\left|B_{n}\right|=A_{n}^{o(1)}$ as $n \rightarrow \infty$;

(iv) $D_{n}=A_{n}^{o(1)}$ as $n \rightarrow \infty$.

Then, the irrationality exponent of the number

$$
\omega=\sum_{n=1}^{\infty} \frac{B_{n}}{A_{n}}
$$

is equal to $\tau$.

Before establishing Lemma 3 we point out that the Folding Lemma remains true if the components of the continued fraction are positive real numbers.

In what follows, for any finite or infinite continued fraction $\zeta=\left[a_{0} ; a_{1}, a_{2}, \ldots\right]$ whose components are real numbers, we define $p_{k}=p_{k}(\zeta)$ and $q_{k}=q_{k}(\zeta)$ as usual by

$$
p_{0}=a_{0}, \quad q_{0}=1, \quad p_{1}=a_{0} a_{1}+1, \quad q_{1}=a_{1},
$$

and

$$
p_{k}=a_{k} p_{k-1}+p_{k-2}, \quad q_{k}=a_{k} q_{k-1}+q_{k-2} \quad \text { for } k \geq 2
$$


Lemma $\mathbf{F}^{\prime}$. Let $\zeta=\left[a_{0} ; a_{1}, \ldots, a_{n}\right]$ be a continued fraction whose components $a_{k}$ are all real numbers with $a_{k} \geq 1$ for $k=1, \ldots, n$. For any real number $t$ with $t \geq 1$ we have

$$
\zeta+\frac{(-1)^{n}}{t q_{n}^{2}}=\left[a_{0} ; a_{1}, a_{2}, \ldots, a_{n-1}, a_{n}, t-1,1, a_{n}-1, a_{n-1}, \ldots, a_{1}\right]=: \zeta_{+}
$$

and

$$
\zeta-\frac{(-1)^{n}}{t q_{n}^{2}}=\left[a_{0} ; a_{1}, a_{2}, \ldots, a_{n-1}, a_{n}-1,1, t-1, a_{n}, a_{n-1}, \ldots, a_{1}\right]=: \zeta_{-} .
$$

Furthermore, we have $q_{2 n+2}\left(\zeta_{+}\right)=q_{2 n+2}\left(\zeta_{-}\right)=t q_{n}^{2}$.

Lemma F' was established by Shallit [25] when the components are integers, but his proof remains valid for the continued fractions as stated above. It is understood that

$$
\left[a_{0} ; a_{1}, \ldots, a_{k}, 1,0, a_{k+1}, a_{k+2}, \ldots\right]=\left[a_{0} ; a_{1}, \ldots, a_{k}, a_{k+1}+1, a_{k+2}, \ldots\right] .
$$

Proof of Lemma 3. We may assume that $S_{0}:=A_{1} / B_{1} \geq 3$ and that $S_{n} \geq 2$ for all $n \geq 1$. Set

$$
\omega_{k}:=\sum_{\nu=1}^{k} \frac{B_{\nu}}{A_{\nu}}
$$

for $k \geq 1$. Starting from $\omega_{1}=\left[0 ; S_{0}\right]$ we have by Lemma $\mathrm{F}^{\prime}$,

$$
\omega_{2}=\left[0 ; S_{0}-1,1, S_{1}-1, S_{0}\right]
$$

or

$$
\omega_{2}=\left[0 ; S_{0}, S_{1}-1,1, S_{0}-1\right]
$$

according as the sign of $B_{2}$ is plus or minus. Then, for $k \geq 2$, we have inductively by Lemma F' again,

$$
\omega_{k+1}=\left[0 ; a_{1}^{\prime}, \ldots, a_{e(k)}^{\prime}, S_{k}-1,1, a_{e(k)}^{\prime}-1, a_{e(k)-1}^{\prime}, \ldots, a_{1}^{\prime}\right]
$$

or

$$
\omega_{k+1}=\left[0 ; a_{1}^{\prime}, \ldots, a_{e(k)}^{\prime}-1,1, S_{k}-1, a_{e(k)}^{\prime}, a_{e(k)-1}^{\prime}, \ldots, a_{1}^{\prime}\right]
$$

according as the sign of $B_{k+1}$ is plus or minus, where

$$
\omega_{k}=\left[0 ; a_{1}^{\prime}, \ldots, a_{e(k)}^{\prime}\right], \quad e(k)=3 \cdot 2^{k-1}-2 .
$$

Thus we get a continued fraction expansion

$$
\omega=\left[0 ; a_{1}, a_{2}, \ldots, a_{n}, \ldots\right]
$$

as the limit of the above expansion of $\omega_{k}$ 's, where the $a_{i}$ 's are rational numbers. We can analyse rational approximations to $\omega$ from this expansion. To this end we summarize several notations and properties concerning this expansion. 
For $n \geq 1$, let $p_{n}=p_{n}(\omega), q_{n}=q_{n}(\omega)$, and observe that we have

$$
q_{n} p_{n-1}-p_{n} q_{n-1}=(-1)^{n}
$$

and

$$
\frac{1}{\left(q_{n+1}+q_{n}\right) q_{n}}<\left|\omega-\frac{p_{n}}{q_{n}}\right|<\frac{1}{q_{n+1} q_{n}} .
$$

We set $p_{n} / q_{n}=p_{n}^{\prime} / q_{n}^{\prime}$, where $p_{n}^{\prime}$ and $q_{n}^{\prime}$ are relatively prime positive integers. It is important to notice that

$$
q_{n}^{\prime}=q_{n}^{1+o(1)}
$$

as $n \rightarrow \infty$. To show this, under the assumption $n \geq 2$, let $k$ be the integer such that $e(k) \leq n<e(k+1)$. It follows from the construction of $\omega_{k}$ and the definition of $D_{k}$ that $D_{k} p_{m}$ and $D_{k} q_{m}$ are integers for $m \leq n$. This with (4.1) implies that the greatest common divisor of $D_{k} p_{n}$ and $D_{k} q_{n}$ is at most $D_{k}^{2}$. Hence, we have $D_{k} q_{n} \geq q_{n}^{\prime} \geq D_{k}^{-1} q_{n}$, which with (iv) implies (4.3).

We now prove that $\mu(\omega) \geq \tau$. To this end we set, for $k \geq 1$,

$$
P_{k}=p_{e(k)}, Q_{k}=q_{e(k)} \quad \text { or } \quad P_{k}=p_{e(k)+1}, Q_{k}=q_{e(k)+1}
$$

according as the sign of $B_{k+1}$ is plus or minus. Then we have

$$
Q_{k+1}=S_{k} Q_{k}^{2}=\frac{A_{k+1}}{\left|B_{k+1}\right|}
$$

by Lemma F', and

$$
\frac{1}{\left(S_{k}+1\right) Q_{k}^{2}}<\left|\omega-\frac{P_{k}}{Q_{k}}\right|<\frac{1}{\left(S_{k}-1\right) Q_{k}^{2}}
$$

by (4.2). Let $\varepsilon>0$ be given. It follows from (ii) that $A_{k+1} \geq A_{k}^{\tau-\varepsilon}$ holds for infinitely many indices $k$. Hence (4.5) together with (4.4) and (iii) implies that

$$
0<\left|\omega-\frac{P_{k}}{Q_{k}}\right|<\frac{1}{Q_{k}^{\tau-2 \varepsilon}}
$$

holds for infinitely many $k$. Since $\varepsilon$ can be taken arbitrarily small, we deduce that $\mu(\omega) \geq \tau$ from (4.3).

We next prove the upper bound $\mu(\omega) \leq \tau$. In the sequel let $\varepsilon>0$ be given, and denote by $n_{j}(\varepsilon)$ with $1 \leq j \leq 5$ positive integers depending only on $\varepsilon$. Let $p / q$ be any reduced fraction with sufficiently large $q$. Assume first that $p / q=p_{n} / q_{n}$ for some $n$. Then we have

$$
\left|\omega-\frac{p}{q}\right|>\frac{1}{3 a_{n+1} q_{n}^{2}}
$$

by $(4.2)$. Let $k$ be the integer such that $e(k) \leq n<e(k+1)$. Since

$$
a_{n+1} \leq \max \left\{a_{e(k)}, \ldots, a_{e(k+1)}\right\} \leq \max \left\{S_{0}, \ldots, S_{k}\right\}
$$


we deduce $a_{n+1} \leq A_{k}^{\tau-2+\varepsilon}$ from the definition of $S_{k}$, (ii) and (iii) provided that $n \geq n_{1}(\varepsilon$ ). Since $Q_{k}=q_{e(k)}$ or $Q_{k}=q_{e(k)}+q_{e(k)-1}$ according as the sign of $B_{k+1}$ is plus or minus, we have $q_{e(k)} \geq Q_{k} / 2$. Hence $q_{n} \geq A_{k} /\left(2\left|B_{k}\right|\right)$ by (4.4). Thus, on noting (iii), we get $a_{n+1} \leq q_{n}^{\tau-2+2 \varepsilon}$ provided that $n \geq n_{2}(\varepsilon)$. Combined with (4.3) and (4.6), this implies

$$
\left|\omega-\frac{p}{q}\right|>\frac{1}{q^{\tau+3 \varepsilon}}
$$

provided that $q \geq n_{3}(\varepsilon)$.

Assume next that $p / q \neq p_{n} / q_{n}$ for all $n$. It follows from (4.2) that

$$
\left|\omega-\frac{p}{q}\right| \geq\left|\frac{p_{n}}{q_{n}}-\frac{p}{q}\right|-\left|\omega-\frac{p_{n}}{q_{n}}\right| \geq \frac{1}{q_{n}^{\prime} q}-\frac{1}{q_{n+1} q_{n}}
$$

for any $n$. We now take $n$ such that $q_{n}^{1-\varepsilon} / 2 \leq q<q_{n+1}^{1-\varepsilon} / 2$. Since $q_{n}^{\prime} \leq q_{n}^{1+\varepsilon}$ by (4.3) provided that $n \geq n_{4}(\varepsilon)$, we have $q_{n}^{\prime} q \leq q_{n}^{1+\varepsilon} q_{n+1}^{1-\varepsilon} / 2 \leq q_{n} q_{n+1} / 2$. Hence we get

$$
\left|\omega-\frac{p}{q}\right| \geq \frac{1}{2 q_{n}^{\prime} q} \geq \frac{1}{2 q(2 q)^{1 /(1-\varepsilon)}}
$$

provided that $q \geq n_{5}(\varepsilon)$. Combined with (4.7), this implies that $\mu(\omega) \leq \tau$, since $\varepsilon$ can be taken arbitrarily small. This completes the proof of the lemma.

\section{On explicit constructions: Mahler's numbers}

In 1937 Mahler [19, 20] established that the Champernowne number

$$
\xi_{\mathbf{c}}=0.1234567891011121314 \ldots,
$$

whose sequence of decimals is given by the concatenation of the integers ranged by increasing order, is a transcendental number. This is a particular case of a more general result; further extensions of which have been given in [21, 26, 27].

The key lemma for Mahler's approach is the following.

Lemma 4. Let $b \geq 2$ be an integer. Let $a$ and $n$ be positive integers. Let $W_{b, n, a}$ be the integer, written in base $b$, whose digits are the concatenation of a copies of every integer having exactly $n$ digits in base $b$. Then, $W_{b, n, a}$ has exactly

$$
D_{b, n, a}:=n a b^{n-1}(b-1)
$$

digits, and

$$
W_{b, n, a}=\frac{b^{n a+n-1}-b^{n-1}+1}{\left(b^{n}-1\right)\left(b^{n a}-1\right)} b^{D_{b, n, a}}-\frac{b^{n a+n}-b^{n}+1}{\left(b^{n}-1\right)\left(b^{n a}-1\right)} .
$$

Lemma 4 is established along the lines of [21], see on page 722 . 
We stress that in the statement of Lemma 4 the base $b$ is not assumed to be in $\mathcal{B}$. This will also be the case throughout this section.

The important fact in Lemma 4 is that all the integers occurring in (5.1), except $b^{D_{b, n, a}}$, are much smaller than $b^{D_{b, n, a}}$. Consequently, the Champernowne number $\xi_{\mathbf{c}}$ can be expressed as a lacunary sum

$$
\sum_{n \geq 1} \frac{c_{n}}{10^{g(n)}}
$$

where $g$ is a rapidly increasing integer valued function and $c_{n}$ is a rational number whose denominator and numerator are small compared with $10^{g(n)}$.

Throughout this section, we use capital letters $W, X, \ldots$ to denote finite words over the alphabet $\{0,1, \ldots, b-1\}$, that we may also view as rational integers, like in Lemmas 4 and 5. This should not cause any confusion. Furthermore, since the proof of Theorem 2 is slightly technical, we prefer to begin with establishing several partial results before explaining how to get a complete proof.

Definition 2. Let $b \geq 2$ be an integer. For a function a taking positive integer values, let $\xi_{b, \mathbf{a}}$ be the real number whose b-ary expansion is given by

$$
\xi_{b, \mathbf{a}}=0 . W_{b, 1, \mathbf{a}(1)} W_{b, 2, \mathbf{a}(2)} \ldots W_{b, n, \mathbf{a}(n)} \ldots
$$

By means of his version of the Folding Lemma, Amou [2] established that

$$
v_{1}\left(\xi_{b, \mathbf{1}}\right)=b-1, \quad \text { for every } b \geq 2,
$$

where 1 denotes the constant function equal to 1 .

Using Lemma 3, we are able to extend this result and construct real numbers with prescribed irrationality exponents. We begin with a particular case of Theorem 2 .

Theorem 6. Let $b \geq 2$ be an integer. For any real number $v \geq b-1$, there exist uncountably many real numbers $\xi$ such that

$$
v_{1}(\xi)=v \quad \text { and } \quad v_{b}(\xi)=v_{b}^{\prime}(\xi)=0
$$

Proof. Let $\tau \geq 1$ be a real number. Consider the function a defined by $\mathbf{a}(n)=\left\lfloor\tau^{n}\right\rfloor$ for $n \geq 1$. For $a \geq 1$ and $n \geq 1$, set

$$
c_{1}(b, n, a)=\frac{b^{n a+n-1}-b^{n-1}+1}{\left(b^{n}-1\right)\left(b^{n a}-1\right)} \quad \text { and } \quad c_{2}(b, n, a)=\frac{b^{n a+n}-b^{n}+1}{\left(b^{n}-1\right)\left(b^{n a}-1\right)},
$$

and observe that, in view of Lemma 4 , the real number $\xi_{b, \mathbf{a}}$ can be written as a lacunary series, namely

$$
\xi_{b, \mathbf{a}}=c_{1}(b, 1, \mathbf{a}(1))+\sum_{n \geq 1} \frac{c_{1}(b, n+1, \mathbf{a}(n+1))-c_{2}(b, n, \mathbf{a}(n))}{b^{\sum_{k=1}^{n} D_{b, k, \mathbf{a}(k)}}} .
$$

For $n \geq 1$, set

$$
A_{n}:=b^{\sum_{k=1}^{n} D_{b, k, \mathbf{a}(k)}}
$$


and

$$
B_{n}:=c_{1}(b, n+1, \mathbf{a}(n+1))-c_{2}(b, n, \mathbf{a}(n)) .
$$

Since $B_{n}$ is very small compared to $A_{n}$ for $n \geq 1$, and

$$
\frac{\sum_{k=1}^{n+1} D_{b, k, \mathbf{a}(k)}}{\sum_{k=1}^{n} D_{b, k, \mathbf{a}(k)}} \rightarrow \tau b,
$$

Lemma 3 can be applied to $\xi_{b, \mathbf{a}}-c_{1}(b, 1, \mathbf{a}(1))$ and it yields that

$$
v_{1}\left(\xi_{b, \mathbf{a}}\right)=\tau b-1
$$

Furthermore, by construction, there are no too long repetitions that occur too early in the $b$-ary expansion of $\xi$, hence, $v_{b}(\xi)=v_{b}^{\prime}(\xi)=0$. This proves the theorem.

Theorem 6 is quite restrictive because of the assumption $v \geq b-1$. For the remaining values of $v$, we need a modified version of Lemma 4 , which incorporates the ideas from [20]. Namely, instead of concatenating integers in some non-decreasing order, we take an integer polynomial $f$ and concatenate the integers $f(j)$. We have the following analogue of Lemma 4, whose proof is implicitly given in [20].

Lemma 5. Let $b \geq 2$ be an integer. Let $a, m$ and $n$ be positive integers. Let $W_{b, n, a, m}$ be the integer, written in base $b$, whose digits are the concatenation of a copies of every $m$-th power having exactly $n$ digits in base $b$. Then, $W_{b, n, a, m}$ has

$$
D_{b, n, a, m}^{\prime} \asymp n a b^{n / m}
$$

digits, and

$$
W_{b, n, a, m}=c_{1}(b, n, a, m) b^{D_{b, n, a, m}^{\prime}}-c_{2}(b, n, a, m),
$$

where $c_{1}(b, n, a, m)$ and $c_{2}(b, n, a, m)$ are rational numbers whose denominators and numerators are $\ll b^{a n(m+1)}$.

Like in Definition 2, for a given function a and a given positive integer $m$, we define numbers $\xi_{b, \mathbf{a}, m}$ by

$$
\xi_{b, \mathbf{a}, m}=0 . W_{b, 1, \mathbf{a}(1), m} W_{b, 2, \mathbf{a}(2), m} \ldots W_{b, n, \mathbf{a}(n), m} \cdots
$$

We choose again the function a defined by $\mathbf{a}(n)=\left\lfloor\tau^{n}\right\rfloor$, for a real number $\tau \geq 1$. It is clear from Lemma 3 that assumption (iii) of Lemma 3 is satisfied. As for assumption $(i v)$, a rapid calculation shows that it holds as soon as $b^{1 / m} \geq 2$. We then infer from Lemma 3 that

$$
v_{1}\left(\xi_{b, \mathbf{a}, m}\right)=\tau b^{1 / m}-1
$$

Selecting for $m$ the largest integer such that $b^{1 / m} \geq 2$, we establish Theorem 2 for $v_{b}=$ $v_{b}^{\prime}=0$ and $v_{1} \geq b^{1 / m}-1$.

To complete the proof of Theorem 2 for $v_{b}=v_{b}^{\prime}=0$, the trick consists in working in the base $b^{t}$, where $t$ is an arbitrarily large integer. Indeed, recall that almost all real 
numbers $\xi$ satisfy $v_{b}(\xi)=v_{b}^{\prime}(\xi)=0$ and $v_{1}(\xi)=1$, and let $v$ be a real number with $v>1$. There exist positive coprime integers $t$ and $m$ such that $2<\left(b^{t}\right)^{1 / m}<v+1$. Choosing now $\tau=(v+1)\left(b^{t}\right)^{-1 / m}$, the above construction shows that

$$
v_{1}\left(\xi_{b^{t}, \mathbf{a}, m}\right)=\tau b^{t / m}-1=v, \quad v_{b^{t}}\left(\xi_{b^{t}, \mathbf{a}, m}\right)=v_{b^{t}}^{\prime}\left(\xi_{b^{t}, \mathbf{a}, m}\right)=0 .
$$

We conclude by using (1.2).

The complete proof of Theorem 2 follows the same general lines as that of Theorem 6 . Besides Lemma 5, the new ingredient is that we insert large blocks of 0 and of 10 between the words $W_{b, n, \mathbf{a}(n), m}$.

Definition 3. Let $b \geq 2$ be an integer. For a positive integer $n$, denote by $X_{n}$ the word composed of $n$ digits 0 and denote by $Y_{n}$ the word composed of the concatenation of $n$ copies of the block 10. Viewed as an integer, we have

$$
Y_{n}=\frac{b^{2 n+1}-b}{b^{2}-1}=\frac{1}{b^{2}-1} \cdot b^{2 n+1}-\frac{b}{b^{2}-1} .
$$

For the same function a as above and for a positive integer $m$, we consider real numbers whose $b$-ary expansions are given by

$$
\xi_{b, \mathbf{n}, \mathbf{s}, \mathbf{a}, m}=0 . W_{b, n_{1}, \mathbf{a}\left(n_{1}\right), m} X_{s_{1}} W_{b, n_{2}, \mathbf{a}\left(n_{2}\right), m} Y_{s_{2}} W_{b, n_{3}, \mathbf{a}\left(n_{3}\right), m} X_{s_{3}} \ldots,
$$

for integer sequences $\mathbf{n}=\left(n_{j}\right)_{j \geq 1}$, and $\mathbf{s}=\left(s_{j}\right)_{j \geq 1}$.

For $j \geq 1$, denote by $r_{j}$ the length of the word $W_{b, n_{j}, \mathbf{a}\left(n_{j}\right), m}$ occurring in the definition of $\xi$, and let $\left(u_{j}\right)_{j \geq 1}$ be the sequence $r_{1}, r_{1}+s_{1}, r_{1}+s_{1}+r_{2}, r_{1}+s_{1}+r_{2}+2 s_{2}, \ldots$ In view of Lemma 5 , there is a sequence $\left(c_{j}\right)_{j \geq 0}$ of rational numbers such that

$$
\xi=c_{0}+\sum_{j \geq 1} \frac{c_{j}}{b^{u_{j}}} .
$$

Furthermore, it follows from Lemma 5 and Definition 3 that the denominator and numerator of $c_{j}$ are small compared with $b^{u_{j}}$. Lemma 3 is then applicable provided that there exists a positive real number $\eta$ such that

$$
u_{j+1} \geq(2+\eta) u_{j}, \quad \text { for all } j \geq 1
$$

This explains why we need to assume that $v_{b}$ and $v_{b}^{\prime}$ are both at least equal to 1 (to settle the case where $v_{b}=0$ and $v_{b}^{\prime} \geq 1$, we simply choose $s_{2 j+1}=0$ for every $j \geq 0$.) This, however, is not enough to deal with the case $v_{1}=1$. Fortunately, (5.2) can be slightly weakened: there exists a sequence $\left(c_{j}\right)_{j \geq 1}$ satisfying $\liminf c_{j}=2$ and $c_{j}>2$ for $j \geq 1$, and such that the property $u_{j+1}=c_{j} u_{j}$ is sufficient to apply Lemma 3 . We omit the technical details, which come from assumption ( $i v)$ of Lemma 3.

Observe furthermore that

$$
v_{b}(\xi)=\limsup _{j \rightarrow+\infty} \frac{u_{4 j+2}}{u_{4 j+1}}
$$




$$
v_{b}^{\prime}(\xi)=\max \left\{v_{b}(\xi), \limsup _{j \rightarrow+\infty} \frac{u_{4 j}}{u_{4 j-1}}\right\}
$$

and

$$
v_{1}(\xi)=\limsup _{j \rightarrow+\infty} \frac{u_{j+1}}{u_{j}},
$$

by Lemma 3. To justify (5.3), we simply note that, by construction, there are no too long blocks of zero occurring near the beginning of the $b$-ary expansion of $\xi$, except the blocks $X_{s_{2 j+1}}$. Likewise, to justify (5.4), we note that, by construction, there are no too long repetitions of a same block occurring near the beginning of the $b$-ary expansion of $\xi$, except those corresponding to the blocks $X_{s_{2 j+1}}$ and $Y_{s_{2 j}}$. Consequently, and since our construction is flexible enough, for any choice of $v_{1}, v_{b}$ and $v_{b}^{\prime}$ as in the statement of Theorem 2, we can find uncountably many suitable choices for the sequences $\left(n_{j}\right)_{j \geq 1}$ and $\left(s_{j}\right)_{j \geq 1}$ such that the corresponding real number $\xi$ satisfies $v_{1}(\xi)=v_{1}, v_{b}(\xi)=v_{b}$ and $v_{b}^{\prime}(\xi)=v_{b}^{\prime}$.

\section{Further results on Problem 2}

Our Theorem 2 is not powerful enough to give a complete answer to Problem 2. However, we are able to treat some of the remaining cases by combining Mahler's constructions with the naïve approach, namely, triangle inequalities. Consequently, in all the

results stated below, we are not able to remove the assumption $v_{1} \geq(1+\sqrt{5}) / 2$, which is crucial for the argument based on triangle inequalities.

We keep the notation of the end of Section 5. We set

$$
\xi_{b, \mathbf{n}, \mathbf{s}, \mathbf{a}, m}=0 . W_{b, n_{1}, \mathbf{a}\left(n_{1}\right), m} X_{s_{1}} W_{b, n_{2}, \mathbf{a}\left(n_{2}\right), m} Y_{s_{2}} W_{b, n_{3}, \mathbf{a}\left(n_{3}\right), m} X_{s_{3}} \ldots,
$$

for integer sequences $\mathbf{n}=\left(n_{j}\right)_{j \geq 1}$, and $\mathbf{s}=\left(s_{j}\right)_{j \geq 1}$. Again, by Lemma 5 , there are a sequence $\left(c_{j}\right)_{j \geq 0}$ of rational numbers and an increasing sequence $\left(u_{j}\right)_{j \geq 1}$ of integers such that

$$
\xi_{b, \mathbf{n}, \mathbf{s}, \mathbf{a}, m}=c_{0}+\sum_{j \geq 1} \frac{c_{j}}{b^{u_{j}}}
$$

For $J \geq 1$, define

$$
\xi_{J}=\xi_{b, \mathbf{n}, \mathbf{s}, \mathbf{a}, m, J}=c_{0}+\sum_{1 \leq j \leq J} \frac{c_{j}}{b^{u_{j}}}=: \frac{p_{J}}{q_{J}}
$$

Proof of Theorem 3. For simplicity, write $\xi$ instead of $\xi_{b, \mathbf{n}, \mathbf{s}, \mathbf{a}, m}$. Let $v \geq 1$ and $w \geq 2$ be real numbers with $w \geq v$. Assume that the parameters occurring in the definition of $\xi$ are chosen in such a way that

$$
\lim _{j \rightarrow+\infty} \frac{u_{2 j+1}}{u_{2 j}}=w \quad \text { and } \quad \lim _{j \rightarrow+\infty} \frac{u_{2 j}}{u_{2 j-1}} \leq v .
$$

Assume further that we are in the following situation:

$$
\left|\xi-p_{2 J} / q_{2 J}\right| \asymp q_{2 J+1}^{-1} \asymp q_{2 J}^{-w}, \quad\left|\xi-p_{2 J+1} / q_{2 J+1}\right| \gg q_{2 J+1}^{-v}, \quad\left|\xi-p_{2 J+2} / q_{2 J+2}\right| \asymp q_{2 J+2}^{-w},
$$


and

$$
q_{2 J+1} \asymp q_{2 J}^{w}, \quad q_{2 J+2} \ll q_{2 J+1}^{v} .
$$

Take a rational $p / q$ with $q_{2 J}<q<q_{2 J+2}$. Using triangle inequalities, we have

$$
|\xi-p / q| \gg q^{-w}
$$

when

$$
q \ll q_{2 J}^{w-1}
$$

Likewise, using that

$$
|\xi-p / q| \gg\left|1 /\left(q q_{2 J+2}\right)-1 / q_{2 J+2}^{w}\right|
$$

we have (6.2) as soon as

$$
q_{2 J+2}^{1 /(w-1)} \ll q \ll q_{2 J+2}^{w-1} .
$$

Recall that, by assumption,

$$
q_{2 J+2} \ll q_{2 J}^{v w}
$$

To get (6.2) for any $q$ between $q_{2 J}$ and $q_{2 J+2}$, we need that $q_{2 J}^{w-1} \gg q_{2 J+2}^{1 /(w-1)}$. This is satisfied as soon as we have

$$
w^{2}-(v+2) w+1 \geq 0
$$

or, equivalently,

$$
w \geq\left(v+2+\sqrt{v^{2}+4 v}\right) / 2 .
$$

Selecting $w=v_{1}+1$ and $v=v_{b}^{\prime}+1$, and choosing $\left(u_{j}\right)_{j \geq 1}$ such that

$$
\lim _{j \rightarrow+\infty} \frac{u_{4 j+2}}{u_{4 j+1}}=v_{b}+1 \quad \text { and } \quad \lim _{j \rightarrow+\infty} \frac{u_{4 j}}{u_{4 j-1}}=v_{b}^{\prime}+1
$$

we check that $v_{1}(\xi)=v_{1}, v_{b}(\xi)=v_{b}$ and $v_{b}^{\prime}(\xi)=v_{b}^{\prime}$. This completes the proof of the theorem.

In the above proof of Theorem 3, we have only used the rational numbers $p_{2 J} / q_{2 J}$ and $p_{2 J+2} / q_{2 J+2}$. If $v$ is not too small, we can do a little better by taking also $p_{2 J+1} / q_{2 J+1}$ into account.

Indeed, using $p_{2 J+1} / q_{2 J+1}$ and triangle inequality, we get (6.2) for $q$ such that

$$
q_{2 J+1}^{1 /(w-1)} \ll q \ll q_{2 J+1}^{v-1}
$$

Clearly, this can be used only if $v$ is not too small, since $v-1 \geq 1 /(w-1)$ is required.

This can be combined with (6.3) if $q_{2 J}^{w-1} \gg q_{2 J+1}^{1 /(w-1)} \asymp q_{2 J}^{w /(w-1)}$, that is, under the assumption that $w \geq(3+\sqrt{5}) / 2$. It then remains to deal with $q$ such that $q_{2 J+1}^{v-1} \ll q \ll$ $q_{2 J+2}$. Combined with (6.4), we see that one needs

$$
q_{2 J+1}^{v-1} \gg q_{2 J+2}^{1 /(w-1)}
$$


By (6.1), this is achieved if

$$
w \geq 2+1 /(v-1),
$$

a weaker assumption than in Theorem 3 when $v$ is not too small.

\section{Metric results}

Throughout this section, we denote by dim the Hausdorff dimension. A classical result, established independently by Jarník [14] and Besicovitch [4], asserts that

$$
\operatorname{dim}\left\{\xi: v_{1}(\xi) \geq v\right\}=\frac{2}{v+1}, \quad \text { for } v \geq 1
$$

Furthermore, for $b \geq 2$, it follows from a general result of Borosh and Fraenkel [5] that

$$
\operatorname{dim}\left\{\xi: v_{b}(\xi) \geq v\right\}=\frac{1}{v+1}, \quad \text { for } v \geq 0 .
$$

For both assertions, the upper bound for the dimension is an easy consequence of the Cantelli lemma, and to establish that the upper bound is actually the exact value of the dimension requires some work.

To get metrical results for sets of real numbers $\xi$ with prescribed values for $v_{b}(\xi)$ for all positive integers $b$, the only available tool is the theory of intersective sets, introduced by Falconer $[12,13]$ and continued by Bugeaud [8] and Durand [11]. We take the notation from [11] with some simplification.

Let $U$ be an open real interval. Let $\left(x_{i}\right)_{i \geq 1}$ be points in $U$ and $\left(r_{i}\right)_{i \geq 1}$ be a sequence of positive real numbers that tends to zero as $i$ tends to infinity. Then, the family $\left(x_{i}, r_{i}\right)_{i \geq 1}$ is a homogeneous ubiquitous system in $U$ if the set

$$
\left\{x \in U:\left|x-x_{i}\right|<r_{i} \quad \text { for i.m. } i \geq 1\right\}
$$

has full Lebesgue measure in $U$. Here and below, we write 'i.m.' for 'infinitely many'.

Let $g: \mathbf{R}_{>0} \rightarrow \mathbf{R}_{>0}$ be non-decreasing on a neighbourhood of 0 and such that $\lim _{x \rightarrow 0} g(x)=0$ and $x \mapsto g(x) / x$ is positive and non-increasing on a neighbourhood of 0 . For any nonempty open real set $V$, Durand [11] defines the class $G^{g}(V)$ composed of the sets with large intersection in $V$. We refer to [11] for a precise definition, and content ourselves to stress that the class $G^{g}(V)$ is stable by countable intersection and that a lower bound for the Hausdorff dimension of any element of $G^{g}(V)$ can be given in terms of the growth of the function $g$. In particular, Durand [11] establishes the following result.

Theorem D. Let $\tau$ be a real number with $\tau \geq 1$ and let $g$ be the function $x \mapsto x^{1 / \tau}$. With the above notation, if the family $\left(x_{i}, r_{i}\right)_{i \geq 1}$ is a homogeneous ubiquitous system in $U$, then the set

$$
\left\{x \in U:\left|x-x_{i}\right|<r_{i}^{\tau} \quad \text { for i.m. } i \geq 1\right\}
$$

belongs to the class $G^{g}(U)$. In particular, its Hausdorff dimension is at least equal to $1 / \tau$.

Since every irrational number can be approximated at order (at least) two by rationals, the family $\left(p / q, q^{-2}\right)$, where $p / q$ runs over all the rational numbers of $(0,1)$, is a 
homogeneous ubiquitous system in $(0,1)$. Then, the difficult half of (7.1) easily follows from Theorem D.

Furthermore, for $b \in \mathcal{B}$, the family $\left(p / q, q^{-1}\right)$, where $p / q$ runs over all the rational numbers of $(0,1)$ with $q$ being a power of $b$, is as well a homogeneous ubiquitous system in $(0,1)$. Again, the difficult half of (7.2) easily follows from Theorem D.

Since the class $G^{g}(V)$ is stable by countable intersection, we get from the above discussion that

$$
\operatorname{dim}\left(\left\{\xi: v_{1}(\xi) \geq 2 v+1\right\} \cap \bigcap_{b \in \mathcal{B}}\left\{\xi: v_{b}(\xi) \geq v\right\}\right)=\frac{1}{v+1}
$$

Using (7.1), (7.2) and standard arguments (like the Hausdorff-Cantelli lemma), we deduce from (7.3) our first result.

Theorem 7. For any non-negative real number $v$, we have

$$
\operatorname{dim}\left(\left\{\xi: v_{1}(\xi)=2 v+1\right\} \cap \bigcap_{b \in \mathcal{B}}\left\{\xi: v_{b}(\xi)=v\right\}\right)=\frac{1}{v+1} .
$$

Since (7.2) also holds with the exponent $v_{b}$ replaced by $v_{b}^{\prime}$, the theory of intersective sets yields the following, stronger result.

Theorem 8. For any non-negative real number $v$, we have

$$
\operatorname{dim}\left(\left\{\xi: v_{1}(\xi)=2 v+1\right\} \cap \bigcap_{b \in \mathcal{B}}\left\{\xi: v_{b}(\xi)=v\right\} \cap \bigcap_{b \in \mathcal{B}}\left\{\xi: v_{b}^{\prime}(\xi)=v\right\}\right)=\frac{1}{v+1}
$$

We choose not to give detailled proofs of Theorems 7 and 8 , since they are easy applications of the theory of intersective sets, which is not the main purpose of the present note.

Clearly, Theorem 4 follows from (1.2) and Theorem 8.

\section{Normal numbers with prescribed irrationality exponent}

Let $b \geq 2$ be an integer. By definition, a real number $\xi$ is normal in base $b$ if, for every positive integer $n$, every block of $n$ digits occurs with the same frequency in the $b$-ary expansion of $\xi$. It follows from the Borel-Cantelli lemma that almost all real numbers $\xi$ are normal in every integer base and satisfy $v_{1}(\xi)=1$.

It was established in [6] that there exist uncountably many Liouville numbers (recall that a Liouville number $\xi$ is a real number with $v_{1}(\xi)=+\infty$ ) that are normal in every integer base. Furthermore, by combining [17] (resp. [16]) with [10], we get that there exist real numbers with arbitrarily large, finite irrationality exponent (resp. badly approximable real numbers) that are normal in every integer base. These statements are obtained by means of metric arguments; consequently, they do not provide us with explicit examples of real numbers with the required properties. Furthermore, to prove that there exist real numbers with arbitrary irrationality exponent that are normal in every integer base remains an open problem; see Problem 33 from [7].

Combining the Folding Lemma with a result of Bailey and Crandall [3], we are able to give a partial, positive answer to this question. 
Theorem 9. Let $b \geq 2$ be an integer and let $v \geq 1$ be a real number. There exist real numbers $\xi$ that are normal in base $b$ and satisfy $v_{1}(\xi)=v$.

This complements the main result of [9], which implies that, for every base $b \geq 2$, there exist real numbers with arbitrary irrationality exponent that are not normal in base $b$.

We stress that the proof of Theorem 9 is constructive. It relies on the following result of Bailey and Crandall [3].

Theorem BC. Let $b \geq 2$ and $c \geq 2$ be coprime integers. Let $\left(m_{k}\right)_{k \geq 1}$ and $\left(n_{k}\right)_{k \geq 1}$ be strictly increasing sequences of positive integers. Assume that the sequence $\left(n_{k}-n_{k-1}\right)_{k \geq 2}$ is non-decreasing and there exists a constant $\gamma>1 / 2$ such that

$$
\frac{m_{k}-m_{k-1}}{c^{\gamma n_{k}}} \geq \frac{m_{k-1}-m_{k-2}}{c^{\gamma n_{k-1}}},
$$

for sufficiently large $k$. Then the real number

$$
\alpha_{b, c, m, n}=\sum_{k \geq 1} \frac{1}{b^{m_{k}} c^{n_{k}}}
$$

is normal in base $b$.

Proof of Theorem 9. Let $b \geq 3$ be an integer. We apply Theorem BC with $c=2$. Let $v>1$ be given. For $k \geq 1$, set $m_{k}=\left\lfloor(1+v)^{k}\right\rfloor$ and $n_{k}=k$. The assumption of Theorem $\mathrm{BC}$ is then satisfied with $\gamma=2 / 3$ and we conclude that the real number

$$
\xi_{v, b}:=\sum_{k \geq 1} \frac{1}{b\left\lfloor(1+v)^{k}\right\rfloor 2^{k}}
$$

is normal in base $b$. It follows from Lemma 3 that $v_{1}\left(\xi_{v, b}\right)=v$.

Likewise, the real number

$$
\xi_{v, 2}:=\sum_{k \geq 1} \frac{1}{2^{\left\lfloor(1+v)^{k}\right\rfloor 3^{k}}}
$$

is normal in base 2 and it satisfies $v_{1}\left(\xi_{v, 2}\right)=v$.

To settle the case $v=1$, we can either invoke the metric theory (see the first paragraph of this section) or proceed as above with $m_{k}=k 2^{k}$ instead of $m_{k}=2^{k}$, in order to be on the safe side for applying Lemma 3.

To conclude this section, we point out that a real number $\xi$ satisfying $v_{b}(\xi)>0$ for some integer $b \geq 2$ cannot be normal in base $b$, for at least one among the digits 0 and $b-1$ does not occur with frequency $1 / b$ in its $b$-ary expansion. It then follows from Theorem 1 that, for any real number $v \geq(1+\sqrt{5}) / 2$, there exist real numbers $\xi$ with $v_{1}(\xi)=v$ that are normal to no integer base $b \geq 2$. This complements a result of Martin [22]. 
Acknowledgements. We are grateful to Arnaud Durand for his comments on Section 7.

\section{References}

[1] B. Adamczewski and Y. Bugeaud, Dynamics for beta-shifts and Diophantine approximation, Ergod. Theory and Dynamical Syst. 27 (2007), 1695-1711.

[2] M. Amou, Approximation to certain transcendental decimal fractions by algebraic numbers, J. Number Theory 37 (1991), 231-241.

[3] D. H. Bailey and R. E. Crandall, Random Generators and Normal Numbers, Experimental Math. 11 (2002), 527-546.

[4] A. S. Besicovitch, Sets of fractional dimension (IV): on rational approximation to real numbers, J. London Math. Soc. 9 (1934), 126-131.

[5] I. Borosh and A. S. Fraenkel, A generalization of Jarnik's theorem on Diophantine approximations, Indag. Math. 34 (1972), 193-201.

[6] Y. Bugeaud, Nombres de Liouville et nombres normaux, C. R. Acad. Sci. Paris, Ser. I 335 (2002), 117-120.

[7] Y. Bugeaud, Approximation by algebraic numbers, Cambridge Tracts in Mathematics, Cambridge, 2004.

[8] Y. Bugeaud, Intersective sets and Diophantine approximation, Mich. Math. J. 52 (2004), 667-682.

[9] Y. Bugeaud, Diophantine approximation and Cantor sets, Math. Ann. 341 (2008), $677-684$.

[10] H. Davenport, P. Erdős and W. J. LeVeque, On Weyl's criterion for uniform distribution, Michigan Math. J. 10 (1963), 311-314.

[11] A. Durand, Sets with large intersection and ubiquity, Math. Proc. Cambridge Philos. Soc. 144 (2008), 119-144.

[12] K. Falconer, Classes of sets with large intersections, Mathematika 32 (1985), 191205.

[13] K. Falconer, Sets with large intersection properties, J. London Math. Soc. 49 (1994), $267-280$.

[14] V. Jarník, Diophantischen Approximationen und Hausdorffsches Mass, Mat. Sbornik 36 (1929), 371-382.

[15] V. Jarník, Über die simultanen Diophantische Approximationen, Math. Z. 33 (1931), $505-543$.

[16] R. Kaufman, Continued fractions and Fourier transforms, Mathematika 27 (1980), $262-267$. 
[17] R. Kaufman, On the theorem of Jarnik and Besicovitch, Acta Arith. 39 (1981), $265-267$.

[18] J. Levesley, C. Salp and S. L. Velani, On a problem of K. Mahler: Diophantine approximation and Cantor sets, Math. Ann. 338 (2007), 97-118.

[19] K. Mahler, Über die Dezimalbruchentwicklung gewisser Irrationalzahlen, Mathematica 4B (Zutphen) (1937), 15 pp.

[20] K. Mahler, Arithmetische Eigenschaften einer Klasse von Dezimalbrüchen, Proc. Akad. v. Wetensch. 40 (1937), 421-428.

[21] K. Mahler, On a Class of Transcendental Decimal Fractions, Comm. Math. Pure Appl. Math. 29 (1976), 717-725.

[22] G. Martin, Absolutely abnormal numbers, Amer. Math. Monthly 108 (2001), 746754 .

[23] M. Mendès France, Sur les fractions continues limitées, Acta Arith. 23 (1973), 207215.

[24] J. O. Shallit, Simple continued fractions for some irrational numbers, J. Number Theory 11 (1979), 209-217.

[25] J. O. Shallit, Simple continued fractions for some irrational numbers, II, J. Number Theory 14 (1982), 228-231.

[26] Y. C. Zhu, On a problem of Mahler concerning the transcendence of a decimal fraction, Acta Math. Sinica 24 (1981), 246-253 (in Chinese).

[27] Y. C. Zhu, A note of irrationality measure of Mahler's transcendental fractions, Acta Math. Sinica 43 (2000), 1-8 (in Chinese).

Masaaki Amou

Department of Mathematics

Gunma University

Tenjin-cho 1-5-1, Kiryu 376-8515

JAPAN

e-mail : amou@math.sci.gunma-u.ac.jp

Yann Bugeaud

Université de Strasbourg

U. F. R. de mathématiques

7 , rue René Descartes

67084 STRASBOURG Cedex

FRANCE

e-mail : bugeaud@math.u-strasbg.fr 